\title{
ANÁLISE ELETROMIOGRÁFICA DOS MÚSCULOS DO ABDOME EM EXERCÍCIOS ABDOMINAIS COM E SEM UTILIZAÇÃO DE DIFERENTES APARELHOS: UMA REVISÃO DE LITERATURA
}

\author{
F. H. Silva*, F. C. Gregório*, L. R. Sousa*, F. J. Arantes*, G. C. Sousa* e F. B. Lizardo* \\ *Laboratório de Eletromiografia Cinesiológica da Universidade Federal de Uberlândia, Uberlândia, \\ Brasil. \\ e-mail: fabricio_henrique_oliveira@hotmail.com \\ Introdução
}

Resumo: Vários aparelhos para exercícios abdominais são difundidos no mercado e muito divulgados na prática do treinamento de força, todavia, existem dúvidas acerca da eficácia destes equipamentos em exercícios abdominais. Portanto, objetivou-se analisar as publicações existentes na literatura científica sobre atividade eletromiográfica dos músculos do abdome em exercícios abdominais com e sem a utilização de diferentes aparelhos. O delineamento metodológico deste estudo caracterizou-se por uma revisão sistemática da literatura que abrangeu livros textos clássicos de Anatomia $\mathrm{Hu}-$ mana e artigos nacionais e internacionais referentes ao tema em questão. Após a análise do material pesquisado, conclui-se que o exercício Tradicional supra abdominal é eficaz no recrutamento do músculo reto do abdome como um todo e na redução da atividade dos músculos flexores da coxa e que apesar da maioria dos aparelhos abdominais não apresentarem justificativas suficientes para substituir o exercício tradicional supra abdominal, esta diversidade de equipamentos pode ser útil para variação de um programa de treinamento.

Palavras-chave: Eletromiografia, Saúde, Core.

\begin{abstract}
Several devices for abdominal exercises are widespread in the market and disclosed in the practice of strength training, however, there are doubts about the effectiveness of these devices in abdominal exercises. Therefore, this study aimed to analyze the publications in scientific literature on electromyographic activity of the abdominal muscles abdominal exercises with and without the use of different devices. The methodological design of this study was characterized by a systematic review of the literature covering classic textbooks of Human Anatomy and national and international articles on the topic in question. After the analysis of the researched material, it is concluded that the traditional abdominal above exercise is effective on the rectus belly muscle recruitment as a whole and in reducing the activity of the flexor muscles of the thigh and although most abdominal devices do not provide sufficient justification to replace exercise traditional abdominal above, this diversity of equipment can be useful to change a training program.
\end{abstract}

Keywords: Electromyography, Health, Core.
A prática de exercícios abdominais tem aumentado visando à prevenção e/ou reabilitação de dores na região lombar (lombalgia), a melhoria do rendimento atlético, aumento da resistência e força do tronco durante o desempenho das atividades da vida diária e a crescente busca da estética que é divulgada pela nossa sociedade $[1,2]$.

Nos exercícios abdominais deve ser priorizado o recrutamento dos músculos abdominais (reto do abdome - parte superior [RAS] e parte inferior [RAI], oblíquo externo do abdome [OE], oblíquo interno do abdome [OI] e transverso do abdome [TA]) de forma a minimizar a atividade dos músculos flexores da coxa, como o reto femoral (RF) [3]. O aumento da atividade do músculo RF nos exercícios abdominais provoca maior tração e força de cisalhamento na região lombar da coluna vertebral [4].

Vários aparelhos para exercícios abdominais são difundidos no mercado e muito divulgados na prática do treinamento de força, todavia, existem dúvidas acerca da eficácia destes diferentes equipamentos em exercícios abdominais [5]. Sabendo que a atividade elétrica dos músculos abdominais em determinados exercícios, compõe a base teórica para o direcionamento de programas de prevenção, reabilitação e treinamento esportivo, espera-se que esta revisão possa proporcionar contribuições relevantes e fornecer dados importantes em relação à atividade eletromiográfica dos músculos RAS, RAI, OE, OI e RF, durante exercícios abdominais com e sem a utilização de diferentes aparelhos.

Portanto, o objetivo deste trabalho foi analisar as publicações existentes na literatura científica sobre atividade eletromiográfica dos músculos do abdome e RF em exercícios abdominais com e sem a utilização de diferentes aparelhos, com intuito de verificar quais aparelhos proporcionam maior recrutamento dos músculos abdominais em relação ao exercício tradicional (exercício supra abdominal).

\section{Materiais e métodos}

O delineamento metodológico deste estudo caracterizou-se por uma revisão sistemática da literatura que abrangeu livros textos clássicos de Anatomia Humana e artigos nacionais e internacionais referentes ao tema em questão. Os livros selecionados foram pesquisados na Biblioteca da Universidade Federal de Uberlândia, enquanto que a identificação dos artigos foi 
feita através de busca bibliográfica na base de dados PubMed/MEDLINE (Literatura Internacional em Ciências da Saúde) e BVS - BIREME, referente aos anos de 2000 a 2016.As palavras-chave utilizadas foram: "electromyography" (eletromiografia), "surface electromyography" (eletromiografia de superfície) e "abdominal exercises" (exercícios abdominais), as palavras foram usadas separadamente e de maneira associada.

\section{Resultados e Discussão}

Após a revisão de literatura, as informações foram estruturadas em duas partes principais apresentadas a seguir: 1) Aspectos morfológicos dos músculos RA, OE e RF e 2) Estudos eletromiográficos durante a realização de exercícios abdominais com e sem a utilização de aparelhos.

\section{1- Aspectos morfológicos dos músculos RA, OE e RF}

A parede abdominal anterolateral tem cinco (pares bilaterais) músculos: três músculos planos e dois músculos verticais. Os três músculos planos são o oblíquo externo do abdome (OE), oblíquo interno do abdome (OI) e transverso do abdome (TA), enquanto os dois músculos verticais da parede abdominal anterolateral, contidos na bainha do músculo reto do abdome, são o grande reto do abdome e o pequeno piramidal [6].

O músculo RA é o principal músculo da parede anterior do abdome, caracteriza-se por ser longo, largo, semelhante a uma tira. Este músculo é uma longa faixa muscular que sobe por toda a parede anterior do abdome, a partir do osso púbis (sínfise púbica) até as cartilagens da $5^{\mathrm{a}}, 6^{\mathrm{a}}$ e $7^{\mathrm{a}}$ costelas e processo xifóide do osso esterno, sendo separado de seu homônimo pela linha alba [6].

Em relação a sua ação, o músculo RA atua principalmente puxando o tronco para frente, quando a pelve está fixa (por exemplo: na flexão do tronco em decúbito dorsal com as pernas fixas). A sua ação consiste no levantamento da pelve, toda vez que a caixa torácica estiver fixa (por exemplo, na elevação do tronco em barra fixa); esta ação depende da participação de outros músculos [7].

Em virtude da sua inserção no osso púbico, este músculo desempenha papel de destaque para a postura normal da pelve, portanto é responsável de modo indireto pela curvatura da coluna lombar; quando este músculo é fraco, a pelve se inclina para frente (anteversão), fato que resulta em aumento patológico da curvatura da região lombar da coluna vertebral (lordose). Finalmente, o músculo RA atua na expiração, impelindo o diafragma para cima, e com sua tensão, protege as vísceras abdominais de lesões ou um impacto [7].

Dos três músculos planos da parede abdominal anterolateral, o músculo OE é o maior e mais superficial, originando-se na face externa das $5^{\mathrm{a}} \quad$ a $12^{\mathrm{a}}$ costelas e se inserindo na linha alba, tubérculo púbico e crista ilíaca [6]. A contração bilateral do músculo $\mathrm{OE}$ atua em sinergismo com o músculo RA nos movimentos de flexão do tronco. A contração unilateral resulta em inclinação do tronco ipsilateral ou em rotação do tronco para o lado oposto, ou seja, o músculo $\mathrm{OE}$ direito do abdome leva à rotação para o lado esquerdo e o lado contrário ocorre o mesmo [7].

O músculo RF situa-se no membro inferior e faz parte do músculo Quadríceps Femoral. Este músculo possui duas cabeças de origem no osso do quadril, uma cabeça retilínea na espinha ilíaca ântero-inferior e uma cabeça reflexa na margem superior do acetábulo, e se insere na tuberosidade da tíbia por meio do ligamento patelar [6].

Em vista de suas fixações, o músculo RF é um músculo bi-articular, ou seja, atua nas articulações do Quadril e do Joelho. Com isso, este músculo participa da flexão da coxa e, se a Coxa está fixa, na rotação da pelve para frente (anteversão) e na sua estabilização, além disso, este músculo realiza a extensão vigorosa da perna, como acontece na corrida e no salto. Um aspecto importante do músculo RF é que este apresenta predomínio de fibras musculares de contração rápida, as quais se inserem em ângulo agudo no respectivo tendão, ou seja, este é um músculo de ação rápida no qual o grau de encurtamento é importante no seu caso [7].

\section{2-Estudos eletromiográficos durante a realização de exercícios abdominais com e sem a utilização de aparelhos}

Nelson et al. [8] analisaram a atividade eletromiográfica da parte superior (RAS) e inferior (RAI) do músculo RA no exercício tradicional supra abdominal em comparação com aparelho AbLounge. Os resultados demonstraram que o sinal eletromiográfico do RAS foi significativamente maior no exercício tradicional em comparação ao aparelho AbLounge, todavia, a atividade do RAI também foi maior no exercício tradicional sem apresentar diferenças estatísticas significativas.

Nelson et al. [8] afirmaram que estes resultados sugerem que o exercício tradicional é mais eficaz do que o AbLounge em relação ao recrutamento do músculo RA. Os autores supracitados destacaram que o AbLounge fornece um bom suporte para a coluna cervical devido o menor grau de amplitude de movimento desta região em relação ao exercício tradicional, desta forma, este aparelho pode se tornar uma alternativa viável para indivíduos com patologias da coluna cervical que podem agravar o problema ao tentar fazer o exercício abdominal tradicional. Esse trabalho apresenta lacunas por não analisar a atividade EMG dos músculos oblíquos e RF.

Willardson et al. [5] analisaram e compararam a ativação dos músculos RA, OE, estabilizadores inferiores do abdome e eretor da espinha, durante a execução de três exercícios tradicionais (supra abdominal, prancha lateral e prancha ventral com 2 apoios) em comparação com aparelho AbCircle. Os autores supracitados demonstraram que o músculo RA apresentou maior atividade no exercício supra abdominal em relação ao nível 1 do aparelho AbCircle e no exercício prancha lateral em comparação com os 
níveis 1 e 2 do referido aparelho. Nenhuma outra diferença significativa foi encontrada no recrutamento dos outros músculos comparando o aparelho AbCircle com os exercícios tradicionais.

Willardson et al. [5] concluíram que a musculatura da parede anterior, lateral e posterior do tronco pode ser ativado de forma semelhante ou até em níveis maiores realizando os três exercícios tradicionais em comparação com aparelho AbCircle. Isto foi particularmente evidente para o exercício prancha lateral, o qual provocou significativamente maior atividade do RA em comparação com AbCirclenível 1 e 2 e produziu atividade similar dos outros músculos analisados em todos os níveis do AbCircle. Com relação a atividade muscular, o AbCircle não fornece um estímulo superior em comparação aos exercícios tradicionais realizados com o próprio peso corporal, entretanto, o equipamento pode ser utilizado como uma alternativa para uma maior variação em um programa de treinamento, dependendo das preferências individuais. Esse trabalho apresenta lacunas ao não analisar a atividade EMG dos músculos Flexores da Coxa.

Lizardo et al. [2] analisaram a atividade eletromiográfica dos músculos RAS, RAI e RF durante o exercício abdominal tradicional e com utilização do aparelho $A b$ Swing (níveis principiante e intermediário). Os autores demonstraram que não houve diferenças significativas na atividade eletromiográfica dos músculos RAS e RAI durante o exercício Tradicional e com $A b$ Swing-nível intermediário, no entanto, o aparelho $A b$ Swing - nível principiante produziu significativamente menor atividade elétrica dos músculos RAS e RAI em comparação aos exercícios tradicional e $A b$ Swing - nível intermediário. Em relação ao músculo RF, o exercício com $A b$ Swing - nível intermediário apresentou significativamente maior sinal eletromiográfico em comparação com $A b$ Swing - nível principiante e exercício Tradicional, sendo que este último demonstrou menor valor em todos exercícios.

Os autores supracitados concluíram que os exercícios com $A b$ Swing - níveis principiante e intermediário podem ser escolhidos para treinamento dos músculos abdominais, entretanto, devem ser utilizados com precauções para pessoas que tenham musculatura abdominal fraca e/ou problemas na região lombar devido a grande ativação do músculo RF quando comparado com exercício Tradicional. Este trabalho apresenta lacunas por não avaliar a atividade EMG dos músculos $\mathrm{OE}$ e OI e por não verificar as diferenças no sinal eletromiográfico entre os gêneros.

Bird et al. [9] compararam a atividade elétrica dos músculos RAS, RAI e OE durante o exercício abdominal tradicional e com aparelho $A b$ Slide e demonstraram que na fase concêntrica os músculos $\mathrm{OE}$ e RAI foram significativamente mais ativos no exercício tradicional, enquanto que na fase excêntrica os músculos OE, RAS e RAI foram significativamente mais recrutados no exercício com $A b$ Slide.

Desta forma, Bird et al. [9] destacaram que o aparelho $\mathrm{Ab}$ Slide pode ser considerado uma alternativa viável para variação em um programa de treinamento abdominal, principalmente quando objetiva-se priorizar o recrutamento dos músculos abdominais durante a fase excêntrica, todavia, este dispositivo não tem benefícios suficientes para justificar a substituição do exercício tradicional. Devido à menor ativação concêntrica e maior controle postural necessário na realização do $A b$ Slide, o exercício supra abdominal deve ser o exercício selecionado na fase inicial do treinamento. Deve-se destacar que esse trabalho apresenta lacunas ao não analisar a atividade EMG dos músculos flexores da coxa (ex: reto femoral) e por não apresentar informações sobre a normalização dos dados.

Escamilla et al. [10] analisaram e compararam o sinal eletromiográfico dos músculos RA, OE, OI, RF, Paravertebrais lombares (PV), Peitoral Maior (PM), Tríceps braquial (TB) e Latíssimo do Dorso (LD) durante dois exercícios abdominais tradicionais (supra abdominal e abdominal completo) e sete aparelhos abdominais (Ab Slide, Ab Twister, AbRocker , AbRoller, Ab Doer, Tronco Track, SAM).

Os exercícios analisados ativaram a musculatura abdominal devido a flexão ativa do tronco (supra abdominal, abdominal completo, SAM, AbRoller, Ab Twister, AbRocker e Ab Doer) ou pela resistência a extensão do tronco ( $A b$ Slide e Torso Track). Os autores supracitados demonstraram que os aparelhos $A b$ Slide e Torso Track foram os mais eficazes na ativação dos músculos abdominais e da extremidade superior, minimizando a atividade dos músculos PV e RF. Os exercícios supra abdominal e abdominal completo produziram recrutamento similar nos músculos abdominais, todavia, os exercícios Ab Twister, AbRocker, $S A M, A b$ Doer e abdominal completo exibiram maior atividade do RF, podendo ser um problema para indivíduos com lombalgia devido a alta atividade deste músculo.

Em outro estudo, Petrofsky et al. [11] analisaram e compararam a atividade eletromiográfica dos músculos RA e OE durante o exercício abdominal na posição sentada com aparelho Cross CrunchMachine (carga baixa: yellowband, carga moderada: orangeband e carga alta:redband) e exercício tradicional supra abdominal. Os principais resultados demonstraram que o exercício no aparelho Cross Crunch Machine com carga alta (red band) foi o que apresentou maior atividade eletromiográfica para os músculos RA e OE em relação ao tradicional (1/3 maior para o RA e duas vezes maior para $\mathrm{OE}$ ), enquanto que na carga moderada (orange band) a atividade foi similar e na carga baixa (yellow band) o recrutamento destes músculos foi menor em comparação ao supra abdominal (aproximadamente a metade).

Petrofsky et al. [11] afirmaram que o exercício abdominal na posição sentada pode ser importante para pessoas com excesso de peso ou idosas, devido às dificuldades pela falta de condicionamento físico para realizar exercício no chão, sendo assim, exercícios abdominais na posição sentada pode ser um estímulo 
para esta população. Além disso, segundo os autores supracitados, a utilização deste aparelho proporcionou aos indivíduos uma forma de trabalhar contra uma carga progressiva permitindo um regime de treinamento progressivo para aumentar a força e a resistência muscular, sendo assim, o trabalho com o equipamento foi maior do que poderia ser realizado no abdominal tradicional.

\section{Conclusões}

O exercício Tradicional supra abdominal é eficaz no recrutamento do músculo RA como um todo e na redução da atividade dos músculos flexores da coxa; Aparelhos abdominais são lançados no mercado e muito difundidos na prática do treinamento de força, entretanto, de acordo com a revisão de literatura, apenas os aparelhos como Ab Slide e Torso Track [10] e Cross Crunch Machine [11] produziram atividade eletromiográfica significativamente maior dos músculos abdominais em relação ao exercício supra abdominal.

Apesar da maioria dos aparelhos abdominais não apresentarem justificativas suficientes para substituir o exercício tradicional supra abdominal, esta diversidade de equipamentos pode ser útil para variação de um programa de treinamento, todavia, alguns aparelhos devem ser utilizados com precauções para pessoas que tenham musculatura abdominal fraca e/ou problemas na região lombar devido à grande ativação do músculo RF;

O local e posicionamento dos eletrodos podem ter grande interferência na qualidade do sinal EMG, portanto, devido à falta de padronização de posicionamento dos eletrodos e a utilização de diferentes formas de processamento do sinal eletromiográfico nos diversos trabalhos, pode-se considerar que estas questões são fatores limitantes para a comparação dos resultados em relação aos diferentes estudos, tornando-se difícil afirmar qual aparelho é mais eficaz em relação ao recrutamento dos músculos abdominais em comparação aos outros equipamentos.

\section{Referências}

[1] Kim, Moon-Hwan; OH, Jae-Seop. Effects of performing an abdominal hollowing exercise on trunk muscle activity during curl-up exercise on an unstable surface. Journalofphysicaltherapyscience, v. 27, n. 2, p. 501-503, 2015.

[2] Lizardo, Frederico Balbino et al. Comparative electromyographic analysis of the Rectus Abdominis and Rectus Femoris muscles in abdominal exercises with or without the $\mathrm{AB}$ swing device. BioscienceJournal, v. 25, n. 3, p. 92-103, 2009

[3] Youdas, James W. et al. An electromyographic analysis of the Ab-Slide exercise, abdominal crunch, supine double leg thrust, and side bridge in healthy young adults: implications for rehabilitation professionals. The Journa-
lofStrength\&ConditioningResearch, v. 22, n. 6 , p. 1939-1946, 2008.

[4] Sundstrap, Emil et al. Swiss ball abdominal crunch with added elastic resistance is an effective alternative to training machines. InternationalJournalof Sports PhysicalTherapy, v. 7, n. 4, 2012.

[5] Willardson, Jeffrey M. et al. A comparison of trunk muscle activation: Ab circle vs. traditional modalities. The Journal of Strength\&ConditioningResearch, v. 24, n. 12, p. 3415-3421, 2010.

[6] Moore, K.L.; Dalley, A.F. Anatomia orientada para clínica. 6 ed. Rio de Janeiro: Guanabara Koogan, 2011.

[7] Weineck, J. Anatomia aplicada ao esporte. 18. ed. São Paulo: Manole, 2013.

[8] Nelson, Gail A.; Bent-Forsity, Denise A.; Roopchnd-Martin, Sharmella C. Electromyographic activity of the rectus abdominis during a traditional crunch and the basic jackknife exercise with the $\mathrm{Ab}$ Lounge ${ }^{\mathrm{TM}}$. The Journal of Strength \& Conditioning Research, v. 26, n. 6 , p. 1584-1588, 2012.

[9] Bird, Michael; Fletcher, Kate M.; Koch, Alex J. Electromyographic comparison of the abslide and crunch exercises. The Journal of Strength \& Conditioning Research, v. 20, n. 2, p. 436-440, 2006.

[10] Escamilla, Rafael F. et al. An electromyographic analysis of commercial and common abdominal exercises: implications for rehabilitation and training.Journal of Orthopaedic\& Sports Physical Therapy, v. 36, n. 2, p. 45-57, 2006.

[11] Petrofsky, Jerrold S. et al. Core muscle strengthening on a portable abdominal machine. Journal of Applied Research in Clinical and Experimental Therapeutics, v. 5, n. 3, p. 460, 2005. 